\title{
Grounds Maintenance Workers
}

National Cancer Institute

\section{Source}

National Cancer Institute. Grounds Maintenance Workers. NCI Thesaurus. Code

C122464.

Workers who provide a pleasant outdoor environment by ensuring that the grounds of houses, businesses, and parks are attractive, orderly, and healthy. 